\title{
Approximate Solutions of Fisher's Type Equations with Variable Coefficients
}

\author{
A. H. Bhrawy ${ }^{1,2}$ and M. A. Alghamdi ${ }^{1}$ \\ ${ }^{1}$ Department of Mathematics, Faculty of Science, King Abdulaziz University, Jeddah 21589, Saudi Arabia \\ ${ }^{2}$ Department of Mathematics, Faculty of Science, Beni-Suef University, Beni-Suef 62511, Egypt
}

Correspondence should be addressed to A. H. Bhrawy; alibhrawy@yahoo.co.uk

Received 6 September 2013; Accepted 20 September 2013

Academic Editor: Dumitru Baleanu

Copyright ( 2013 A. H. Bhrawy and M. A. Alghamdi. This is an open access article distributed under the Creative Commons Attribution License, which permits unrestricted use, distribution, and reproduction in any medium, provided the original work is properly cited.

\begin{abstract}
The spectral collocation approximations based on Legendre polynomials are used to compute the numerical solution of timedependent Fisher's type problems. The spatial derivatives are collocated at a Legendre-Gauss-Lobatto interpolation nodes. The proposed method has the advantage of reducing the problem to a system of ordinary differential equations in time. The four-stage A-stable implicit Runge-Kutta scheme is applied to solve the resulted system of first order in time. Numerical results show that the Legendre-Gauss-Lobatto collocation method is of high accuracy and is efficient for solving the Fisher's type equations. Also the results demonstrate that the proposed method is powerful algorithm for solving the nonlinear partial differential equations.
\end{abstract}

\section{Introduction}

Spectral methods (see, for instance, [1-5]) are powerful techniques that we use to numerically solve linear and nonlinear partial differential equations either in their strong or weak forms. What sets spectral methods apart from others like finite difference methods or finite element methods is that to get a spectral method we approximate the solutions by high order orthogonal polynomial expansions. The orthogonal polynomial approximations can have very high convergence rates, which allow us to use fewer degrees of freedom for a desired level of accuracy. The most common spectral method from the strong form of the equations is known as collocation. In collocation techniques, the partial differential equation must be satisfied at a set of grid, or more precisely, collocation points (see, for instance, [6-10]). Spectral methods also have become increasingly popular for solving fractional differential equations [11-21].

In this paper, we present an accurate numerical solution based on Legendre-Gauss-Lobatto collocation method for Fisher's type equations. The Fisher equation in the form

$$
u_{t}=D u_{x x}+v u(1-u)
$$

was firstly introduced by Fisher in [22] to describe the propagation of a mutant gene. Fisher equations have a wide application in a large number of the chemical kinetics [23], logistic population growth [24], flame propagation [25], population in one-dimensional habitual [26], neutron population in a nuclear reaction [27], neurophysiology [28], branching Brownian motion [23], autocatalytic chemical reactions [29], and nuclear reactor theory [30].

In recent years, many physicists and mathematicians have paid much attention to the Fisher equations due to their importance in mathematical physics. In [31], Ögün and Kart utilized truncated Painlevè expansions for presenting some exact solutions of Fisher and generalized Fisher equations. Tan et al. [28] proposed the homotopy analysis method to find analytical solution of Fisher equations. Gunzburger et al. [32] applied the discrete finite element approximation for obtaining a numerical solution of the forced Fisher equation. Dag et al. [33] discussed and applied the B-spline Galerkin method for Fisher's equation. Bastani and Salkuyeh [34] proposed the compact finite difference approach in combination with third-order Runge-Kutta scheme to solve Fisher's equation. More recently, Mittal and Jain [35] investigated the cubic B-spline scheme for solving Fisher's reaction-diffusion problem. However, the fisher equations have been studied in 
many other articles by numerous numerical methods such as pseudospectral method $[36,37]$, finite difference method [38-44], finite element method [45], B-spline algorithm [46], and Galerkin method [47, 48].

To increase the numerical solution accuracy, spectral collocation methods based on orthogonal polynomials are often chosen. Doha et al. [49] proposed and developed a new numerical algorithm for solving the initial-boundary system of nonlinear hyperbolic equations based on spectral collocation method; a Chebyshev-Gauss-Radau collocation method in combination with the implicit Runge-Kutta scheme are employed to obtain highly accurate approximations to this system of nonlinear hyperbolic equations. In [50], Bhrawy proposed an efficient Jacobi-Gauss-Lobatto collocation method for approximating the solution of the generalized Fitzhugh-Nagumo equation in which the JacobiGauss-Lobatto points are used as collocation nodes for spatial derivatives. Moreover, the Jacobi spectral collocation methods are used to solve some problems in mathematical physics, (see, for instance, [51-53]).

Indeed, there are no results on Legendre-Gauss-Lobatto collocation method for solving nonlinear Fisher-type equations subject to initial-boundary conditions. Therefore, the objective of this work is to present a numerical algorithm for solving such equation based on Legendre-Gauss-Lobatto pseudospectral method. The spatial derivatives are approximated at these grid points by approximating the derivatives of Legendre polynomial that interpolates the solutions. Moreover, we set the boundary conditions in the collocation method. The problem is then reduced to system of firstorder ordinary differential equations in time. The fourstage A-stable implicit Runge-Kutta scheme is proposed for treating the this system of equations. Finally, some illustrative examples are implemented to illustrate the efficiency and applicability of the proposed approach.

The rest of this paper is structured as follows. In the next section, some properties of Legendre polynomials, which are required for implementing our algorithm, are presented. Section 3 is devoted to the development of Gauss-Lobatto collocation technique for a general form of Fisher-type equations based on the Legendre polynomials, and in Section 4 the proposed method is implemented to obtain some numerical results for three problems of Fisher-type equations with known exact solutions. Finally, a brief conclusion is provided in Section 5.

\section{Legendre Polynomials}

The Legendre polynomials $L_{k}(x)(k=0,1, \ldots)$ satisfy the following Rodrigues' formula:

$$
L_{k}(x)=\frac{(-1)^{k}}{2^{k} k !} D^{k}\left(\left(1-x^{2}\right)^{k}\right)
$$

we recall also that $L_{k}(x)$ is a polynomial of degree $k$, and therefore, the $q$ th derivative of $L_{k}(x)$ is given by

$$
L_{k}^{(q)}(x)=\sum_{i=0(k+i=\mathrm{even})}^{k-q} C_{q}(k, i) L_{i}(x),
$$

where

$$
\begin{aligned}
& C_{q}(k, i) \\
& \qquad=\frac{2^{q-1}(2 i+1) \Gamma[(q+k-i) / 2] \Gamma[(q+k+i+1) / 2]}{\Gamma[q] \Gamma[(2-q+k-i) / 2] \Gamma[(3-q+k+i) / 2]} .
\end{aligned}
$$

The analytical form of Legendre polynomial is

$$
L_{n}(x)=\sum_{i=0}^{[n / 2]} c_{k}^{(n)} x^{n-2 k}
$$

where $c_{k}^{(n)}=(-1)^{k}(2 n-2 k) ! / 2^{n}(n-k) !(n-2 k) ! k !$, and

$$
\left[\frac{n}{2}\right]= \begin{cases}\frac{n}{2}, & \text { even, } \\ \frac{n-1}{2}, & \text { odd. }\end{cases}
$$

It is also generating from the following relation:

$$
L_{k+2}(x)=\frac{2 k+3}{k+2} x L_{k+1}(x)-\frac{k+1}{k+2} L_{k}(x),
$$

with $L_{0}(x)=1, L_{1}(x)=x$, and satisfies the orthogonality condition

$$
\left(L_{k}(x), L_{l}(x)\right)_{w}=\int_{-1}^{1} L_{k}(x) L_{l}(x) w(x)=h_{k} \delta_{l k} .
$$

where $w(x)=1, h_{k}=2 /(2 k+1)$. Let $S_{N}$ be the space of all polynomials of degree $\leq N$, then for any $\phi \in S_{2 N-1}(0, L)$,

$$
\int_{-1}^{1} w(x) \phi(x) d x=\sum_{j=0}^{N} \omega_{N, j} \phi\left(x_{N, j}\right)
$$

Let us define the following discrete inner product and norm:

$$
(u, v)_{w}=\sum_{j=0}^{N} u\left(x_{N, j}\right) v\left(x_{N, j}\right) \omega_{N, j}
$$

where $x_{N, j}$ and $\omega_{N, j}$ are the nodes and the corresponding weights of the Legendre-Gauss-Lobatto quadrature formula on the interval $(-1,1)$, respectively.

\section{Legendre Spectral Collocation Method}

Because of the pseudospectral method is an efficient and accurate numerical scheme for solving various problems in physical space, including variable coefficient and singularity (see, $[54,55]$ ), we propose this method based on Legendre polynomials for approximating the solution of the nonlinear generalized Burger-Fisher model equation and Fisher model with variable coefficient.

3.1. (1+1)-Dimensional Generalized Burger-Fisher Equation. In this subsection, we derive a Legendre pseudospectral 
algorithm to solve numerically the generalized Burger-Fisher problem:

$$
u_{t}+v u^{\delta} u_{x}-u_{x x}-\gamma u\left(1-u^{\delta}\right)=0, \quad(x, t) \in D \times[0, T]
$$

where $D=\{x:-1 \leq x \leq 1\}$. Subject to

$$
\begin{gathered}
u(x, t)=g(t), \quad x=-1,1, \\
u(x, 0)=f(x), \quad x \in D .
\end{gathered}
$$

In the following, we shall derive an efficient algorithm for the numerical solution of (11)-(13). Let the approximation of $u(x, t)$ be given in terms of the Legendre polynomials expansion:

$$
u(x, t)=\sum_{j=0}^{N} a_{j}(t) L_{j}(x), \quad \mathbf{a}=\left(a_{0}, a_{1}, \ldots, a_{N}\right)^{T}
$$

Making use of relations (8) and (10) gives

$$
u(x, t)=\sum_{j=0}^{N}\left(\frac{1}{h_{j}} \sum_{i=0}^{N} L_{j}\left(x_{i}\right) L_{j}(x) \omega_{N, i} u\left(x_{i}, t\right)\right)
$$

or equivalently

$$
u(x, t)=\sum_{i=0}^{N}\left(\sum_{j=0}^{N} \frac{1}{h_{j}} L_{j}\left(x_{i}\right) L_{j}(x) \omega_{N, i}\right) u\left(x_{i}, t\right)
$$

The Gauss-Lobatto points were introduced by way of (9). We then saw that the polynomial approximation $u(x, t)$ can be characterized by $(N+1)$ nodal values $u\left(x_{i}, t\right)$. The approximation of the spatial partial derivatives of firstorder for $u(x, t)$ can be computed at the Legendre GaussLobatto interpolation nodes as

$$
\begin{aligned}
u_{x}\left(x_{n}, t\right) & =\sum_{i=0}^{N}\left(\sum_{j=0}^{N} \frac{1}{h_{j}} L_{j}\left(x_{i}\right)\left(L_{j}\left(x_{n}\right)\right)^{\prime} \omega_{N, i}\right) u\left(x_{i}, t\right) \\
& =\sum_{i=0}^{N} A_{n i} u\left(x_{i}, t\right) \\
& =\sum_{i=0}^{N} A_{n i} u_{i}(t), \quad n=0,1, \ldots, N
\end{aligned}
$$

where

$$
\begin{gathered}
A_{n i}=\sum_{j=0}^{N} \frac{1}{h_{j}} L_{j}\left(x_{i}\right)\left(L_{j}\left(x_{n}\right)\right)^{\prime} \Phi_{N, i}, \\
u_{i}(t)=u\left(x_{i}, t\right) .
\end{gathered}
$$

Subsequently, the second-order spatial partial derivatives of $u(x, t)$ may be written at the same collocation nodes as

$$
\begin{aligned}
u_{x x}\left(x_{n}, t\right) & =\sum_{i=0}^{N}\left(\sum_{j=0}^{N} \frac{1}{h_{j}} L_{j}\left(x_{i}\right)\left(L_{j}\left(x_{n}\right)\right)^{\prime \prime} \omega_{N, i}\right) u\left(x_{i}, t\right) \\
& =\sum_{i=0}^{N} B_{n i} u\left(x_{i}, t\right) \\
& =\sum_{i=0}^{N} B_{n i} u_{i},
\end{aligned}
$$

where

$$
B_{n i}=\sum_{j=0}^{N} \frac{1}{h_{j}} L_{j}\left(x_{i}\right)\left(L_{j}\left(x_{n}\right)\right)^{\prime \prime} \omega_{N, i}
$$

In collocation methods, one specifically seeks the approximate solution such that the problem (11) is satisfied exactly at the Legendre Gauss-Lobatto set of interpolation points $x_{n}$; $n=1, \ldots, N-1$. The approximation is exact at the $N-$ 1 collocation points. Therefore, (11) after using relations (17)(20), can be written as

$$
\begin{array}{r}
u_{n}(t)+v u_{n}^{\delta}(t) \sum_{i=0}^{N} A_{n i} u_{i}(t) \\
-\sum_{i=0}^{N} B_{n i} u_{i}(t)-\gamma u_{n}(t)\left(1-u_{n}^{\delta}(t)\right)=0, \\
n=1, \ldots, N-1,
\end{array}
$$

where $u_{n}(t)=u\left(x_{n}, t\right)$ and $u_{n}(t)=\partial \dot{u}_{n}(t) / \partial t$.

Now the two values $u_{0}(t)$ and $u_{N}(t)$ can be determined from the boundary conditions (12), then (21) can be reformulated as

$$
\begin{aligned}
& u_{n}(t)+v u_{n}^{\delta}(t) \sum_{i=1}^{N-1} A_{n i} u_{i}(t) \\
& \quad-\sum_{i=1}^{N-1} B_{n i} u_{i}(t)+v u_{n}^{\delta}(t) d_{n}(t)-\widetilde{d_{n}}(t) \\
& \quad-\gamma u_{n}(t)\left(1-u_{n}^{\delta}(t)\right)=0, \quad n=1, \ldots, N-1,
\end{aligned}
$$

where

$$
\begin{aligned}
& d_{n}(t)=A_{n 0} u_{0}(t)+A_{n N} u_{N}(t), \\
& \widetilde{d_{n}}(t)=B_{n 0} u_{0}(t)+B_{n N} u_{N}(t) .
\end{aligned}
$$

Approximation (22) automatically satisfies the boundary conditions (12), but we need an initial condition for each of the $u_{n}(t)$ to integrate (22) in time. The initial condition is usually taken to be the interpolant of the initial 
function $f(x)$; that is $u_{n}(0)=f\left(x_{n}\right)$. Therefore, the approximation of (11)-(13) is reduced to the solution of system of ordinary differential equations in time. Consider

$$
\begin{gathered}
u_{n}(t)+v u_{n}^{\delta}(t) \sum_{i=1}^{N-1} A_{n i} u_{i}(t)-\sum_{i=1}^{N-1} B_{n i} u_{i}(t) \\
+v u_{n}^{\delta}(t) d_{n}(t)-\widetilde{d}_{n}(t)-\gamma u_{n}(t)\left(1-u_{n}^{\delta}(t)\right)=0, \\
n=1, \ldots, N-1, \\
u_{n}(0)=f\left(x_{n}\right) .
\end{gathered}
$$

Let us denote

$$
\begin{gathered}
U^{\cdot}(t)=\left[u_{1}(t), u_{2}(t), \ldots, \dot{u_{N-1}}(t)\right]^{T}, \\
U(0)=\left[u_{1}(0), u_{2}(0), \ldots, u_{N-1}(0)\right]^{T}, \\
f=\left[f\left(x_{1}\right), f\left(x_{2}\right), \ldots, f\left(x_{N-1}\right)\right]^{T}, \\
F(t, u(t))=\left[F_{1}(t, u(t)), F_{2}(t, u(t)), \ldots, F_{N-1}(t, u(t))\right]^{T}, \\
F_{n}(t, u(t))=-v u_{n}^{\delta}(t) \sum_{i=1}^{N-1} A_{n i} u_{i}(t)+\sum_{i=1}^{N-1} B_{n i} u_{i}(t) \\
\quad-v u_{n}^{\delta}(t) d_{n}(t)+\widetilde{d_{n}}(t)+\gamma u_{n}(t)\left(1-u_{n}^{\delta}(t)\right), \\
n=1, \ldots, N-1 .
\end{gathered}
$$

Then (24) can be written in the matrix form

$$
\begin{gathered}
U^{\cdot}(t)=F(t, u(t)) \\
U(0)=f .
\end{gathered}
$$

This system of ordinary differential equations can be solved by using four-stage A-stable implicit Runge-Kutta scheme.

\section{2. (1+1)-Dimensional Fisher Equation with Variable Coef-} ficient. In this subsection, we extend the application of the Legendre pseudospectral method to solve numerically the Fisher equation with variable coefficient,

$$
u_{t}-b(t) u_{x x}-c u(1-u)=0, \quad(x, t) \in D \times[0, T],
$$

subject to the initial-boundary conditions

$$
\begin{gathered}
u(x, 0)=f(x), \quad x \in D, \\
u(x, t)=g(t) .
\end{gathered}
$$

Proceeding as in the previous subsection we can obtain $u_{x x}$ in the same form as (19), and then (27) can be collocated in the Legendre Gauss-Lobatto points as:

$$
\begin{aligned}
u_{n}(t) & -b(t) \sum_{i=1}^{N-1} B_{n i} u_{i}(t)-b(t) \widetilde{d_{n}}(t)-c u_{n}(t)\left(1-u_{n}(t)\right) \\
& =0, \quad n=1, \ldots, N-1
\end{aligned}
$$

TABLE 1: Absolute errors for Example 1.

\begin{tabular}{lccccc}
\hline$x$ & $t$ & $E(x, t)$ & $x$ & $t$ & $E(x, t)$ \\
\hline-1 & & $5.56 \times 10^{-11}$ & -1 & & $8.93 \times 10^{-11}$ \\
-0.5 & & $1.46 \times 10^{-8}$ & -0.5 & & $8.23 \times 10^{-9}$ \\
0 & 0.1 & $1.95 \times 10^{-8}$ & 0 & 0.2 & $1.41 \times 10^{-8}$ \\
0.5 & & $1.55 \times 10^{-8}$ & 0.5 & & $1.17 \times 10^{-8}$ \\
1 & & $5.56 \times 10^{-11}$ & 1 & & $8.93 \times 10^{-11}$ \\
\hline
\end{tabular}

which can be written in the matrix form

$$
U^{\cdot}(t)=F(t, u(t)),
$$$$
U(0)=f \text {, }
$$

where

$$
\begin{aligned}
& F_{n}(t, u(t)) \\
& =b(t) \sum_{i=1}^{N-1} B_{n i} u_{i}(t)+b(t) \widetilde{d_{n}}(t)+c u_{n}(t)\left(1-u_{n}(t)\right), \\
& n=1, \ldots, N-1 .
\end{aligned}
$$

\section{Numerical Examples}

In this section, three nonlinear time-dependent Fisher-type equations on finite interval are implemented to demonstrate the accuracy and capability of the proposed algorithm, and all of them were performed on the computer using a program written in Mathematica 8.0. The absolute errors in the given tables are $E(x, t)=\mid u(x, t)-\tilde{u}(x, t)$ where $u(x, t)$ and $\widetilde{u}(x, t)$ are the exact and numerical solution at selected points $(x, t)$.

Example 1. Consider the nonlinear time-dependent onedimensional Fisher-type equations

$$
u_{t}=u_{x x}+u(1-u)(u-\gamma), \quad(x, t) \in D \times[0, T],
$$

where $D=\{x:-1<x<1\}$. Subject to

$$
\begin{aligned}
& u(1, t)=\frac{1+\gamma}{2}-\frac{\gamma-1}{2} \tanh \left[\frac{\gamma-1}{2 \sqrt{2}}\left(1-\frac{1+\gamma}{\sqrt{2}} t\right)\right], \\
& u(-1, t)=\frac{1+\gamma}{2}+\frac{\gamma-1}{2} \tanh \left[\frac{\gamma-1}{2 \sqrt{2}}\left(1+\frac{1+\gamma}{\sqrt{2}} t\right)\right], \\
& u(x, 0)=\frac{1+\gamma}{2}-\frac{\gamma-1}{2} \tanh \left[\frac{\gamma-1}{2 \sqrt{2}}(x)\right], \quad x \in D .
\end{aligned}
$$

The exact solution is

$$
u(x, t)=\frac{1+\gamma}{2}-\frac{\gamma-1}{2} \tanh \left[\frac{\gamma-1}{2 \sqrt{2}}\left(x-\frac{1+\gamma}{\sqrt{2}} t\right)\right]
$$

In Table 1, we introduce the absolute errors between the approximate and exact solutions for problem (32) using the proposed method for different values of $x$ and $t$, with $\gamma=$ $10^{-2}$ and $N=20$. 


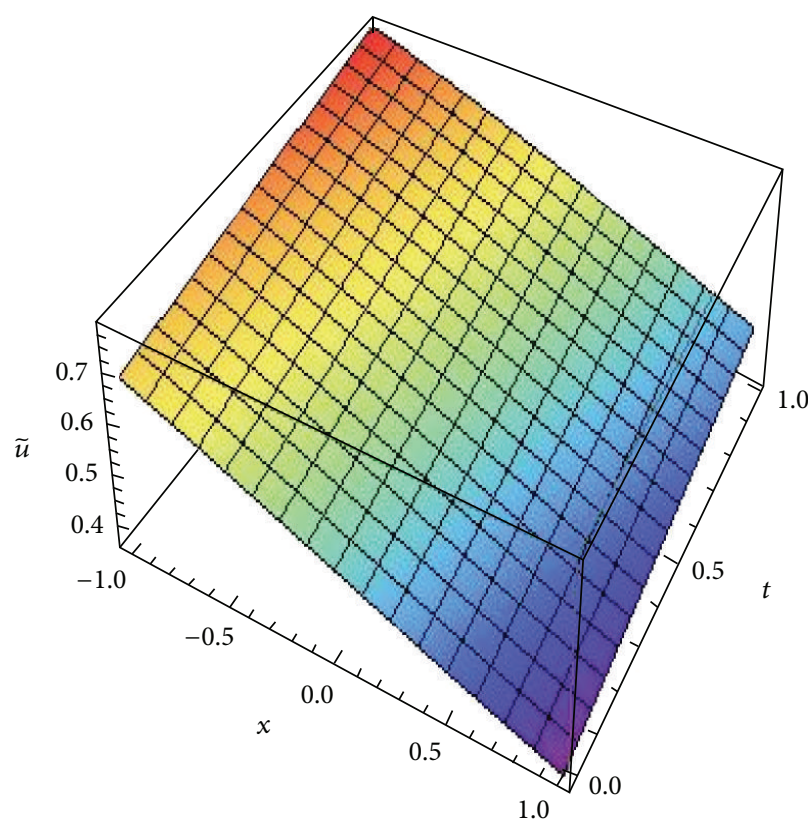

(a)

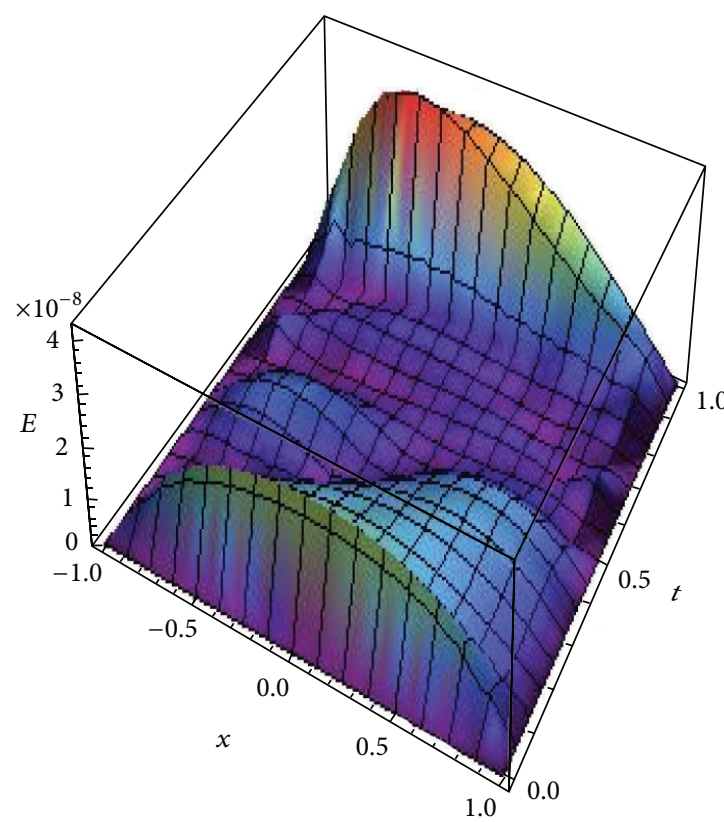

(b)

Figure 1: The result of the L-GL-C method at $\gamma=10^{-2}$ and $N=20$. (a) The approximate solution. (b) The absolute error.

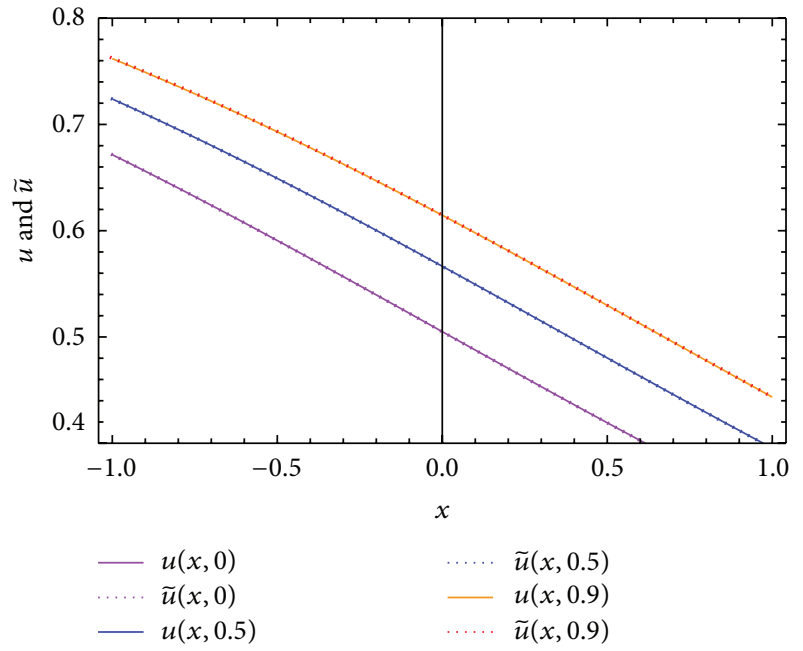

FIGURE 2: The curves of approximate solutions and the exact solutions of problem (32) at $t=0.0, t=0.5$, and $t=0.9$ with $\gamma=$ $10^{-2}$ and $N=20$.

In case of $\gamma=10^{-2}$ and $N=20$, the approximate solution and absolute errors of problem (32) are displayed in Figures 1(a) and 1(b), respectively. In Figure 2, we plotted the curves of approximate solutions and exact solutions of problem (32) for different values of $t(0.0,0.5$ and 0.9) with $\gamma=$ $10^{-2}$ and $N=20$. It is clear from this figure that approximate solutions and exact solutions completely coincide for the chosen values of $t$.
Example 2. Consider the nonlinear time-dependent onedimensional generalized Burger-Fisher-type equations

$$
u_{t}=u_{x x}-v u^{\delta} u_{x}+\gamma u\left(1-u^{\delta}\right), \quad(x, t) \in D \times[0, T]
$$

where $D=\{x:-1<x<1\}$. Subject to

$$
\begin{aligned}
& u(1, t) \\
&=\left(\frac{1}{2}-\frac{1}{2} \tanh \left[\frac{\nu \delta}{2(\delta+1)}\right.\right.\left.\left.\times\left(1-\left(\frac{v}{\delta+1}+\frac{\gamma(\delta+1)}{v}\right) t\right)\right]\right)^{1 / \delta}, \\
& u(-1, t) \quad\left.\left.\times\left(1+\left(\frac{v}{\delta+1}+\frac{\gamma(\delta+1)}{v}\right) t\right)\right]\right)^{1 / \delta}, \\
&=\left(\frac{1}{2}+\frac{1}{2} \tanh \left[\frac{\nu \delta}{2(\delta+1)}\right.\right. \\
& u(x, 0)=\left(\frac{1}{2}-\frac{1}{2} \tanh \left[\frac{\nu \delta}{2(\delta+1)} x\right]\right)^{1 / \delta}, \quad x \in D .
\end{aligned}
$$




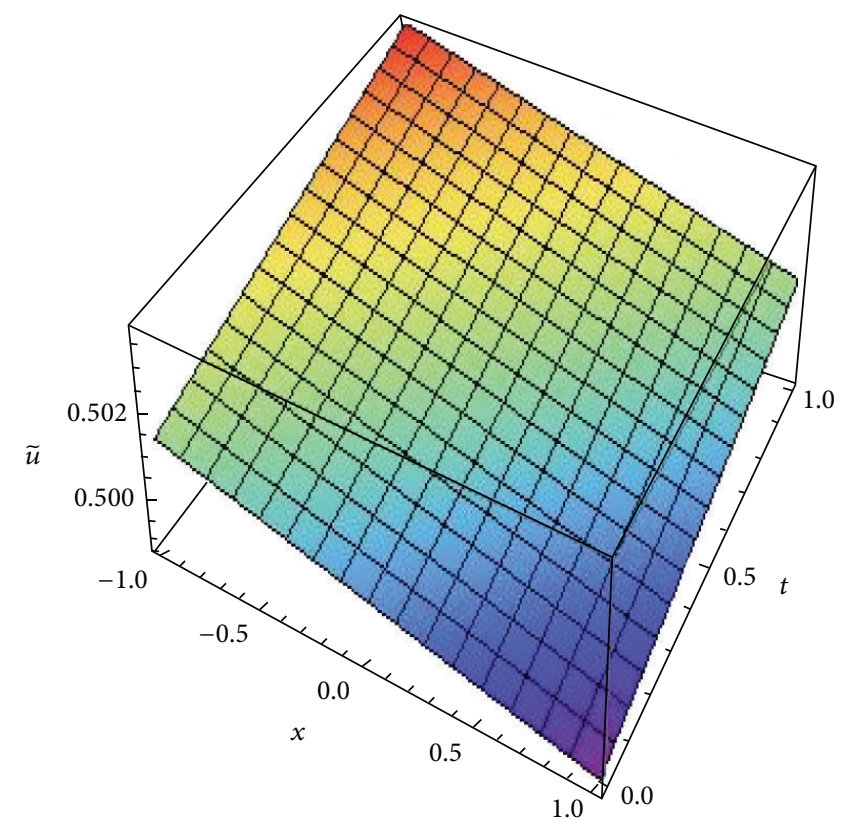

(a)

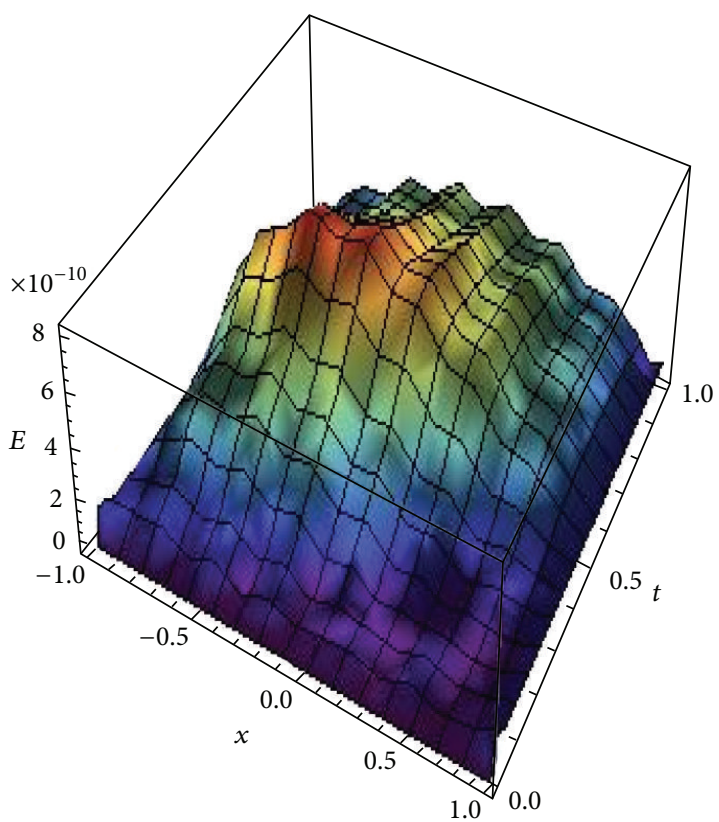

(b)

Figure 3: The result of the L-GL-C method at $\nu=\gamma=10^{-2}, \delta=1$, and $N=20$. (a) The approximate solution. (b)The absolute error.

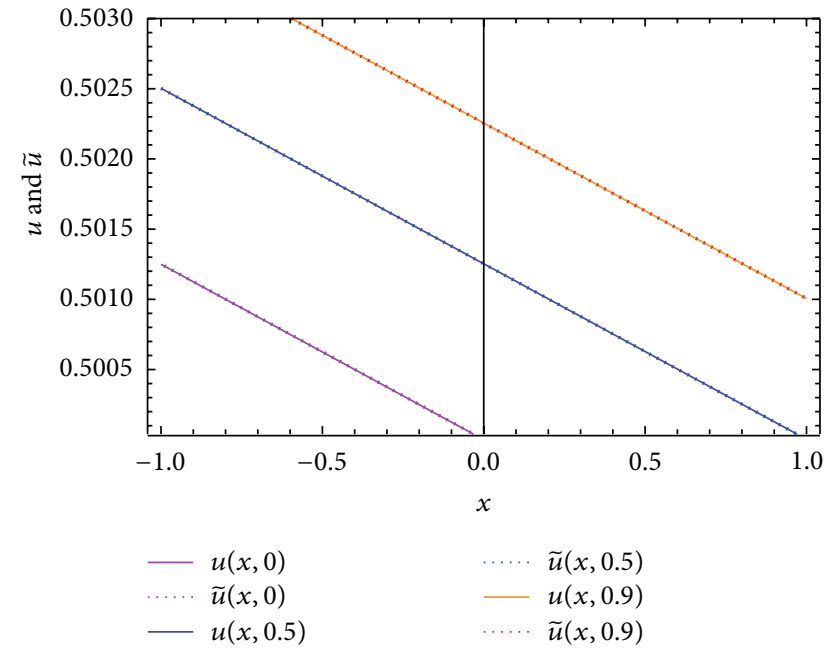

FIGURE 4: The curves of approximate solutions and the exact solutions of problem (35) at $t=0.0, t=0.5$, and $t=0.9$ with $\nu=\gamma=10^{-2}, \delta=1$, and $N=20$.

The exact solution of (35) is

$$
u(x, t)
$$

$$
\begin{aligned}
=\left(\frac{1}{2}-\frac{1}{2} \tanh (\right. & \frac{\nu \delta}{2(\delta+1)} \\
& \left.\left.\times\left(x-\left(\frac{v}{\delta+1}+\frac{\gamma(\delta+1)}{\nu}\right) t\right)\right)\right)^{1 / \delta} .
\end{aligned}
$$

The absolute errors for problem (35) are listed in Table 2 using the L-GL-C method with $v=\gamma=10^{-2}, N=20$, and various choices of $\delta$.

To illustrate the effectiveness of the Legendre pseudospectral method for problem (35), we displayed in Figures 3(a) and 3 (b) the approximate solution and the absolute error with $v=$ $\gamma=10^{-2}, \delta=1$, and $N=20$. The graph of curves of exact and approximate solutions with different values of $t(0.0$, 0.5 , and 0.9 ) is given in Figure 4. Moreover, the approximate solution and the absolute error with $\nu=\gamma=10^{-2}, \delta=2$, and $N=20$ are displayed in Figures 5(a) and 5(b), respectively. The curves of exact and approximate solutions of problem (35) with $\delta=2$ are displayed in Figure 6 with values of parameters listed in its caption.

Example 3. Consider the nonlinear time-dependent onedimensional Fisher-type equations with variable coefficient

$$
\begin{array}{r}
u_{t}=-\frac{a}{6 \mu^{2}} \operatorname{coth}\left(\frac{a}{6} t+c\right) u_{x x}+a u(1-u), \\
(x, t) \in D \times[0, T]
\end{array}
$$

where $D=\{x:-1<x<1\}$. Subject to

$$
\begin{aligned}
u(1, t)= & \frac{1}{4} \operatorname{coth}\left(\frac{a}{6} t+c\right) \operatorname{sech}^{2}\left(\frac{\mu}{2}+\frac{5 a}{12} t\right) \\
& +\frac{1}{2} \tanh \left(\frac{\mu}{2}+\frac{5 a}{12} t\right)+\frac{1}{2},
\end{aligned}
$$




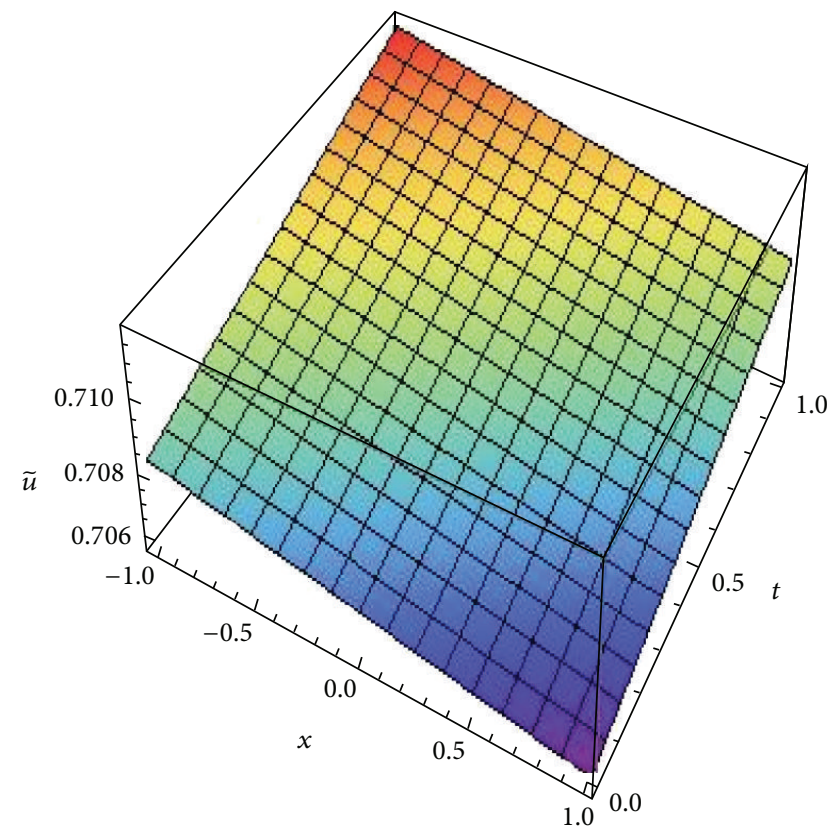

(a)

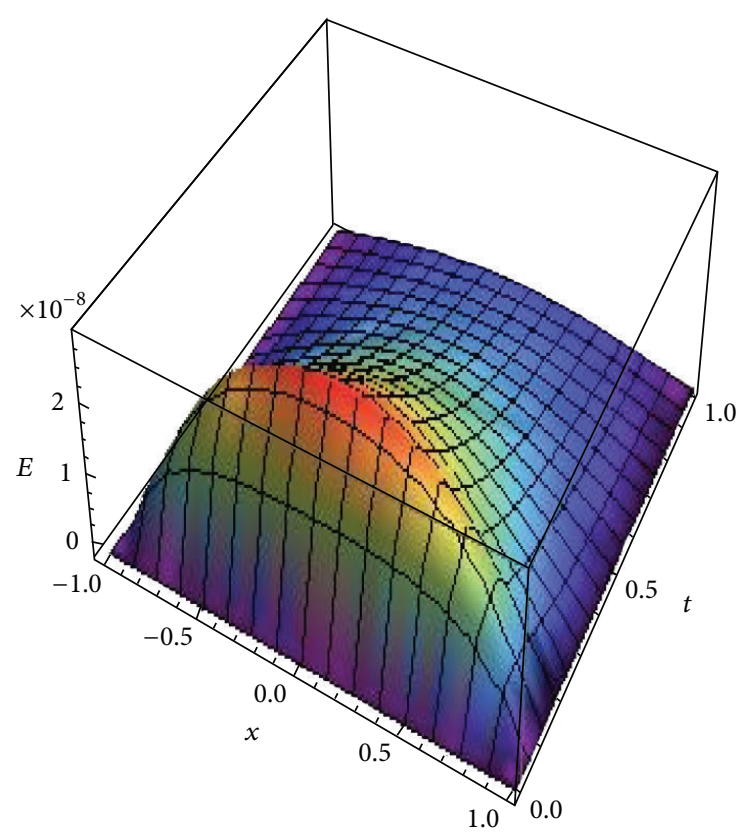

(b)

FIGURE 5: The result of the L-GL-C method at $\nu=\gamma=10^{-2}, \delta=2$, and $N=20$. (a) The approximate solution. (b)The absolute error.

TABle 2: Absolute errors for Example 2.

\begin{tabular}{|c|c|c|c|c|c|c|c|c|}
\hline \multirow[b]{2}{*}{$x$} & \multicolumn{2}{|c|}{$\delta=1$} & \multicolumn{3}{|c|}{$\delta=2$} & \multicolumn{3}{|c|}{$\delta=3$} \\
\hline & $t$ & $E(x, t)$ & $x$ & $t$ & $E(x, t)$ & $x$ & $t$ & $E(x, t)$ \\
\hline-1 & & $8.51 \times 10^{-11}$ & -1 & & $1.26 \times 10^{-10}$ & -1 & & $6.91 \times 10^{-11}$ \\
\hline-0.5 & & $1.16 \times 10^{-10}$ & -0.5 & & $2.34 \times 10^{-8}$ & -0.5 & & $1.09 \times 10^{-8}$ \\
\hline 0 & 0.1 & $5.36 \times 10^{-12}$ & 0 & 0.1 & $2.61 \times 10^{-8}$ & 0 & 0.1 & $1.30 \times 10^{-8}$ \\
\hline 0.5 & & $1.53 \times 10^{-11}$ & 0.5 & & $2.33 \times 10^{-8}$ & 0.5 & & $1.09 \times 10^{-8}$ \\
\hline 1 & & $8.51 \times 10^{-11}$ & 1 & & $1.26 \times 10^{-10}$ & 1 & & $6.91 \times 10^{-11}$ \\
\hline-1 & & $8.71 \times 10^{-11}$ & -1 & & $1.22 \times 10^{-10}$ & -1 & & $1.41 \times 10^{-10}$ \\
\hline-0.5 & & $7.77 \times 10^{-10}$ & -0.5 & & $8.24 \times 10^{-9}$ & -0.5 & & $4.27 \times 10^{-9}$ \\
\hline 0 & 0.5 & $6.49 \times 10^{-10}$ & 0 & 0.5 & $1.16 \times 10^{-8}$ & 0 & 0.5 & $5.89 \times 10^{-9}$ \\
\hline 0.5 & & $3.81 \times 10^{-10}$ & 0.5 & & $8.26 \times 10^{-9}$ & 0.5 & & $4.28 \times 10^{-9}$ \\
\hline 1 & & $8.71 \times 10^{-11}$ & 1 & & $1.22 \times 10^{-10}$ & 1 & & $1.41 \times 10^{-10}$ \\
\hline
\end{tabular}

$$
\begin{aligned}
u(-1, t)= & \frac{1}{4} \operatorname{coth}\left(\frac{a}{6} t+c\right) \operatorname{sech}^{2}\left(-\frac{\mu}{2}+\frac{5 a}{12} t\right) \\
& +\frac{1}{2} \tanh \left(-\frac{\mu}{2}+\frac{5 a}{12} t\right)+\frac{1}{2}, \\
u(x, 0)= & \frac{1}{4} \operatorname{coth}(c) \operatorname{sech}^{2}\left(\frac{\mu x}{2}\right) \\
& +\frac{1}{2} \tanh \left(\frac{\mu x}{2}\right)+\frac{1}{2}, \quad x \in D .
\end{aligned}
$$

The exact solution of $(38)$ is

$$
\begin{aligned}
u(x, t)= & \frac{1}{4} \operatorname{coth}\left(\frac{a}{6} t+c\right) \operatorname{sech}^{2}\left(\frac{\mu x}{2}+\frac{5 a}{12} t\right) \\
& +\frac{1}{2} \tanh \left(\frac{\mu x}{2}+\frac{5 a}{12} t\right)+\frac{1}{2} .
\end{aligned}
$$

Table 3 lists the absolute errors for problem (38) using the L-GL-C method. From numerical results of this table, it can be concluded that the numerical solutions are in excellent agreement with the exact solutions. 


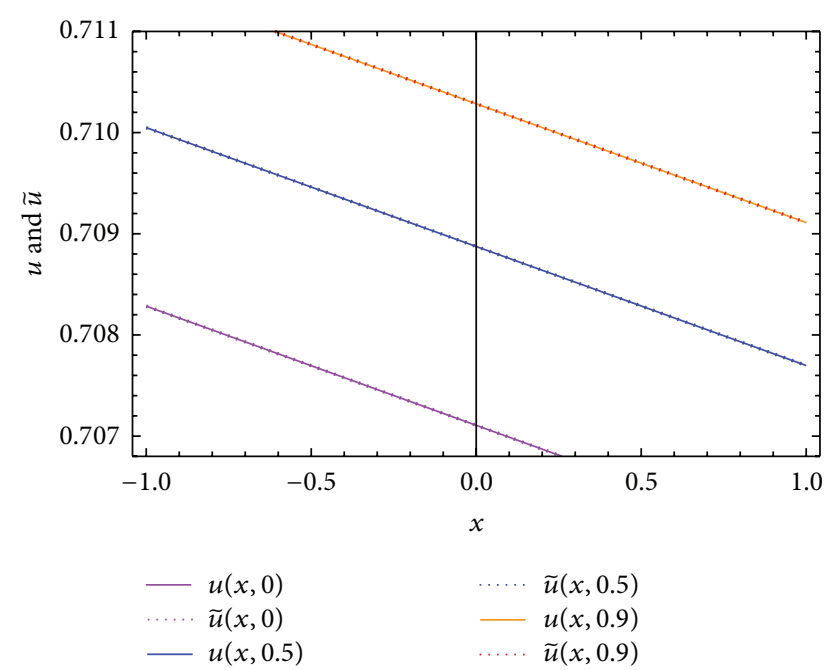

FIGURE 6: The curves of approximate solutions and the exact solutions of problem (35) at $t=0.0, t=0.5$, and $t=0.9$ with $\nu=\gamma=10^{-2}, \delta=2$, and $N=20$.

TABLE 3: Absolute errors for Example 3.

\begin{tabular}{lccccc}
\hline$x$ & $t$ & $E(x, t)$ & $x$ & $t$ & $E(x, t)$ \\
\hline-1 & & $9.81 \times 10^{-10}$ & -1 & & $7.05 \times 10^{-9}$ \\
-0.5 & & $1.00 \times 10^{-7}$ & -0.5 & & $1.93 \times 10^{-6}$ \\
0 & 0.1 & $1.45 \times 10^{-7}$ & 0 & 0.5 & $2.84 \times 10^{-6}$ \\
0.5 & & $1.19 \times 10^{-7}$ & 0.5 & & $2.12 \times 10^{-6}$ \\
1 & & $9.81 \times 10^{-10}$ & 1 & & $7.06 \times 10^{-9}$ \\
\hline
\end{tabular}

\section{Conclusion}

In this paper, based on the Legendre-Gauss-Lobatto pseudospectral approximation we proposed an efficient numerical algorithm to solve nonlinear time-dependent Fisher-type equations with constant and variable coefficients. The method is based upon reducing the nonlinear partial differential equation into a system of first-order ordinary differential equations in the expansion coefficient of the spectral solution. Numerical examples were also provided to illustrate the effectiveness of the derived algorithms. The numerical experiments show that the Legendre pseudospectral approximation is simple and accurate with a limited number of collocation nodes.

\section{Conflict of Interests}

The authors declare that there is no conflict of interests regarding the publication of this paper.

\section{References}

[1] D. A. Kopriva, Implementing Spectral Methods for Partial Differential Equations: Algorithms for Scientists and Engineers, Springer, Berlin, Germany, 2009.

[2] C. Canuto, M. Y. Hussaini, A. Quarteroni, and T. A. Zang, Spectral Methods: Fundamentals in Single Domains, Springer, Berlin, Germany, 2006.
[3] C. I. Gheorghiu, Spectral Methods for Differential Problems, T. Popoviciu Institute of Numerical Analysis,, Cluj-Napoca, Romaina, 2007.

[4] E. H. Doha, W. M. Abd-Elhameed, and A. H. Bhrawy, "New spectral-Galerkin algorithms for direct solution of high evenorder differential equations using symmetric generalized Jacobi polynomials," Collectanea Mathematica, vol. 64, no. 3, pp. 373394, 2013.

[5] E. H. Doha and A. H. Bhrawy, "An efficient direct solver for multidimensional elliptic Robin boundary value problems using a Legendre spectral-Galerkin method," Computers \& Mathematics with Applications, vol. 64, no. 4, pp. 558-571, 2012.

[6] E. H. Doha, A. H. Bhrawy, D. Baleanu, and S. S. Ezz-Eldien, "On shifted Jacobi spectral approximations for solving fractional differential equations," Applied Mathematics and Computation, vol. 219, no. 15, pp. 8042-8056, 2013.

[7] O. R. Isik and M. Sezer, "Bernstein series solution of a class of Lane-Emden type equations," Mathematical Problems in Engineering, Article ID 423797, 9 pages, 2013.

[8] E. Tohidi, A. H. Bhrawy, and K. Erfani, "A collocation method based on Bernoulli operational matrix for numerical solution of generalized pantograph equation," Applied Mathematical Modelling, vol. 37, no. 6, pp. 4283-4294, 2013.

[9] M. S. Mechee and N. Senu, "Numerical study of fractional differential equations of Lane-Emden type by method of collocation," Applied Mathematics, vol. 3, pp. 851-856, 2012.

[10] A. H. Bhrawy and W. M. Abd-Elhameed, "New algorithm for the numerical solutions of nonlinear third-order differential equations using Jacobi-Gauss collocation method," Mathematical Problems in Engineering, vol. 2011, Article ID 837218, 14 pages, 2011.

[11] A. Ahmadian, M. Suleiman, S. Salahshour, and D. Baleanu, "A Jacobi operational matrix for solving a fuzzy linear fractional differential equation," Advances in Difference Equations, vol. 2013, article 104, 29 pages, 2013.

[12] D. Baleanu, A. H. Bhrawy, and T. M. Taha, "Two efficient generalized Laguerre spectral algorithms for fractional initial value problems," Abstract and Applied Analysis, vol. 2013, Article ID 546502, 10 pages, 2013.

[13] D. Baleanu, A. H. Bhrawy, and T. M. Taha, "A modified Generalized Laguerre spectral method for fractional differential equations on the half line," Abstract and Applied Analysis, vol. 2013, Article ID 413529, 12 pages, 2013.

[14] A. Ahmadian, M. Suleiman, and S. Salahshour, "An operational matrix based on Legendre polynomials for solving fuzzy fractional-order differential equations," Abstract and Applied Analysis, vol. 2013, Article ID 505903, 29 pages, 2013.

[15] F. Ghaemi, R. Yunus, A. Ahmadian, S. Salahshourd, M. Suleiman, and S. F. Saleh, "Application of fuzzy fractional kinetic equations to modelling of the acid hydrolysis reaction," Abstract and Applied Analysis, vol. 2013, Article ID 610314, 19 pages, 2013.

[16] M. H. Atabakzadeh, M. H. Akrami, and G. H. Erjaee, "Chebyshev operational matrix method for solving multi-order fractional ordinary differential equations," Applied Mathematical Modelling, vol. 37, no. 20-21, pp. 8903-8911, 2013.

[17] A. H. Bhrawy and M. M. Al-Shomrani, "A shifted Legendre spectral method for fractional-order multi-point boundary value problems," Advances in Difference Equations, vol. 2012, article 8, 19 pages, 2012.

[18] X. Ma and C. Huang, "Spectral collocation method for linear fractional integro-differential equations," Applied Mathematical Modelling, 2013. 
[19] M. R. Eslahchi, M. Dehghan, and M. Parvizi, "Application of the collocation method for solving nonlinear fractional integrodifferential equations," Journal of Computational and Applied Mathematics, vol. 257, pp. 105-128, 2013.

[20] Y. Yangy and Y. Huang, "Spectral-collocation methods for fractional pantograph delay-integrodifferential equations," Advances in Mathematical Physics. In press.

[21] A. H. Bhrawy and A. S. Alofi, "The operational matrix of fractional integration for shifted Chebyshev polynomials," Applied Mathematics Letters, vol. 26, no. 1, pp. 25-31, 2013.

[22] R. A. Fisher, "The wave of advance of advantageous genes," Annals of Eugenics, vol. 7, pp. 335-369, 1937.

[23] A. J. Khattak, "A computational meshless method for the generalized Burger's-Huxley equation," Applied Mathematical Modelling, vol. 33, no. 9, pp. 3718-3729, 2009.

[24] N. F. Britton, Reactiondiffusion Equations and Their Applications to Biology, Academic Press, London, UK, 1986.

[25] D. A. Frank, Diffusion and Heat Exchange in Chemical Kinetics, Princeton University Press, Princeton, NJ, USA, 1955.

[26] A. Wazwaz, "The extended tanh method for abundant solitary wave solutions of nonlinear wave equations," Applied Mathematics and Computation, vol. 187, no. 2, pp. 1131-1142, 2007.

[27] W. Malfliet, "Solitary wave solutions of nonlinear wave equations," American Journal of Physics, vol. 60, no. 7, pp. 650-654, 1992.

[28] Y. Tan, H. Xu, and S.-J. Liao, "Explicit series solution of travelling waves with a front of Fisher equation," Chaos, Solitons and Fractals, vol. 31, no. 2, pp. 462-472, 2007.

[29] H. N. A. Ismail, K. Raslan, and A. A. A. Rabboh, "Adomian decomposition method for Burger's-Huxley and Burger'sFisher equations," Applied Mathematics and Computation, vol. 159, no. 1, pp. 291-301, 2004.

[30] J. Canosa, "Diffusion in nonlinear multiplicative media," Journal of Mathematical Physics, vol. 10, no. 10, pp. 1862-1868, 1969.

[31] A. Öğün and C. Kart, "Exact solutions of Fisher and generalized Fisher equations with variable coefficients," Acta Mathematicae Applicatae Sinica, vol. 23, no. 4, pp. 563-568, 2007.

[32] M. D. Gunzburger, L. S. Hou, and W. Zhu, "Fully discrete finite element approximations of the forced Fisher equation," Journal of Mathematical Analysis and Applications, vol. 313, no. 2, pp. 419-440, 2006.

[33] I. Dag, A. Şahin, and A. Korkmaz, "Numerical investigation of the solution of Fisher's equation via the B-spline galerkin method," Numerical Methods for Partial Differential Equations, vol. 26, no. 6, pp. 1483-1503, 2010.

[34] M. Bastani and D. K. Salkuyeh, "A highly accurate method to solve Fisher's equation," Pramana, vol. 78, no. 3, pp. 335-346, 2012.

[35] R. C. Mittal and R. K. Jain, "Numerical solutions of nonlinear Fisher's reaction-diffusion equation with modified cubic Bspline collocation method," Mathematical Sciences, vol. 7, article $12,2013$.

[36] J. Gazdag and J. Canosa, "Numerical solution of Fisher's equation," Journal of Applied Probability, vol. 11, pp. 445-457, 1974.

[37] T. Zhao, C. Li, Z. Zang, and Y. Wu, "Chebyshev-Legendre pseudo-spectral method for the generalised Burgers-Fisher equation," Applied Mathematical Modelling, vol. 36, no. 3, pp. 1046-1056, 2012.

[38] G. Gürarslan, "Numerical modelling of linear and nonlinear diffusion equations by compact finite difference method,"
Applied Mathematics and Computation, vol. 216, no. 8, pp. $2472-$ 2478,2010 .

[39] X. Y. Chen, Numerical methods for the Burgers-Fisher equation [M.S. thesis], University of Aeronautics and Astronautics, Nanjing, China, 2007.

[40] R. E. Mickens and A. B. Gumel, "Construction and analysis of a non-standard finite difference scheme for the Burgers-Fisher equation," Journal of Sound and Vibration, vol. 257, no. 4, pp. 791-797, 2002.

[41] R. E. Mickens, "A best finite-difference scheme for the Fisher equation," Numerical Methods for Partial Differential Equations, vol. 10, no. 5, pp. 581-585, 1994.

[42] R. E. Mickens, "Relation between the time and space stepsizes in nonstandard finite-difference schemes for the Fisher equation," Numerical Methods for Partial Differential Equations, vol. 15, no. 1, pp. 51-55, 1997.

[43] N. Parekh and S. Puri, "A new numerical scheme for the Fisher equation," Journal of Physics A, vol. 23, no. 21, pp. L1085-L1091, 1990.

[44] R. Rizwan-Uddin, "Comparison of the nodal integral method and nonstandard finite-difference schemes for the Fisher equation," SIAM Journal on Scientific Computing, vol. 22, no. 6, pp. 1926-1942, 2001.

[45] R. Chernma, "Exact and numerical solutions of tiie generalized fisher equation," Reports on Mathematkxl Physics, vol. 47, pp. 393-411, 2001.

[46] A. Şahin, I. Dag, and B. Saka, "A B-spline algorithm for the numerical solution of Fisher's equation," Kybernetes, vol. 37, no. 2, pp. 326-342, 2008.

[47] J. Roessler and H. Hüssner, "Numerical solution of the $1+$ 2 dimensional Fisher's equation by finite elements and the Galerkin method," Mathematical and Computer Modelling, vol. 25, no. 3, pp. 57-67, 1997.

[48] J. Roessler and H. Hüssner, "Numerical solution of the $1+$ 2 dimensional Fisher's equation by finite elements and the Galerkin method," Mathematical and Computer Modelling, vol. 25, no. 3, pp. 57-67, 1997.

[49] E. H. Doha, A. H. Bhrawy, R. M. Hafez, and M. A. Abdelkawy, "A Chebyshev-Gauss-Radau scheme for nonlinear hyperbolic system of first order," Applied Mathematics and Information Science, vol. 8, no. 2, pp. 1-10, 2014.

[50] A. H. Bhrawy, "A Jacobi-Gauss-Lobatto collocation method for solving generalized Fitzhugh-Nagumo equation with timedependent coefficients," Applied Mathematics and Computation, vol. 222, pp. 255-264, 2013.

[51] E. H. Doha, D. Baleanu, A. H. Bhrawy, and M. A. Abdelkawy, "A Jacobi collocation method for solving nonlinear Burgers'-type equations," Abstract and Applied Analysis, vol. 2013, Article ID 760542, 12 pages, 2013.

[52] A. H. Bhrawy, L. M. Assas, and M. A. Alghamdi, "Fast spectral collocation method for solving nonlinear time-delayed Burgers-type equations with positive power terms," Abstract and Applied Analysis, vol. 2013, Article ID 741278, 12 pages, 2013.

[53] A. H. Bhrawy, L. M. Assas, and M. A. Alghamdi, "An efficient spectral collocation algorithm for nonlinear Phi-four equations," Boundary Value Problems, vol. 2013, article 87, 16 pages, 2013.

[54] A. Saadatmandi, "Bernstein operational matrix of fractional derivatives and its applications," Applied Mathematical Modelling, 2013. 
[55] A. H. Bhrawy and A. S. Alofi, "A Jacobi-Gauss collocation method for solving nonlinear Lane-Emden type equations," Communications in Nonlinear Science and Numerical Simulation, vol. 17, no. 1, pp. 62-70, 2012. 


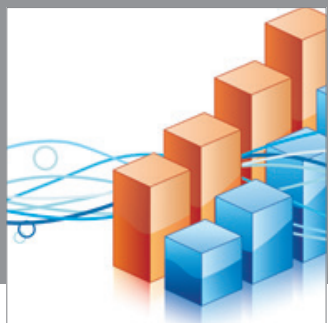

Advances in

Operations Research

mansans

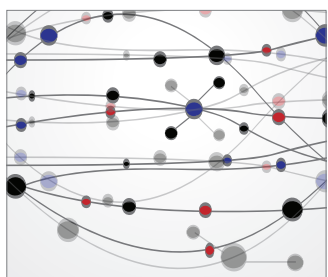

The Scientific World Journal
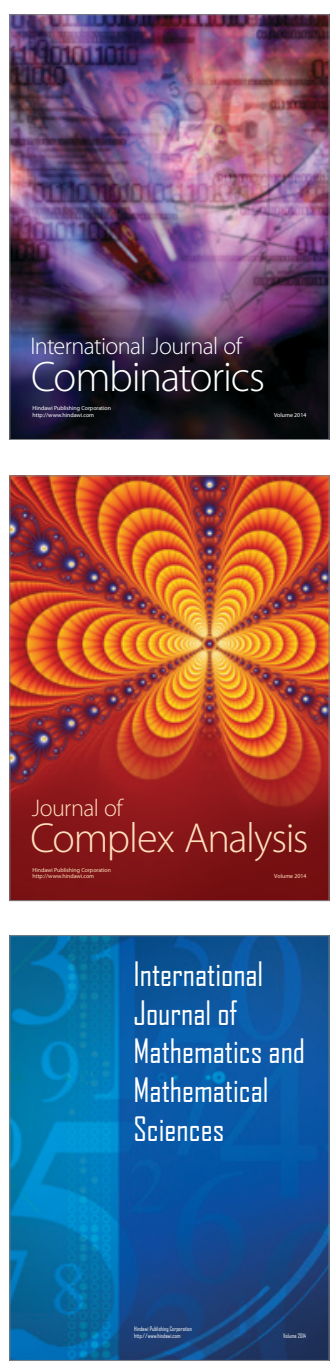
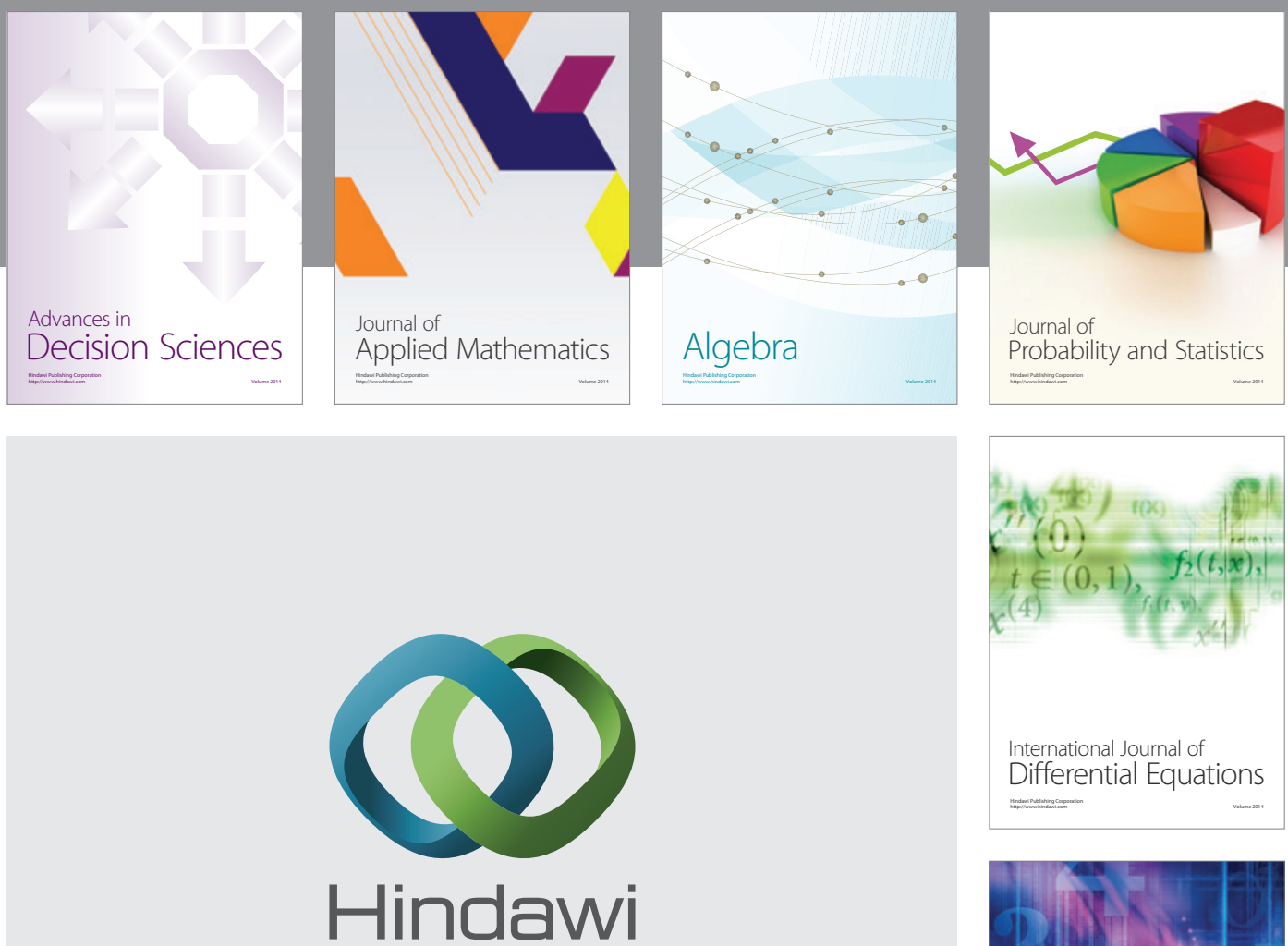

Submit your manuscripts at http://www.hindawi.com
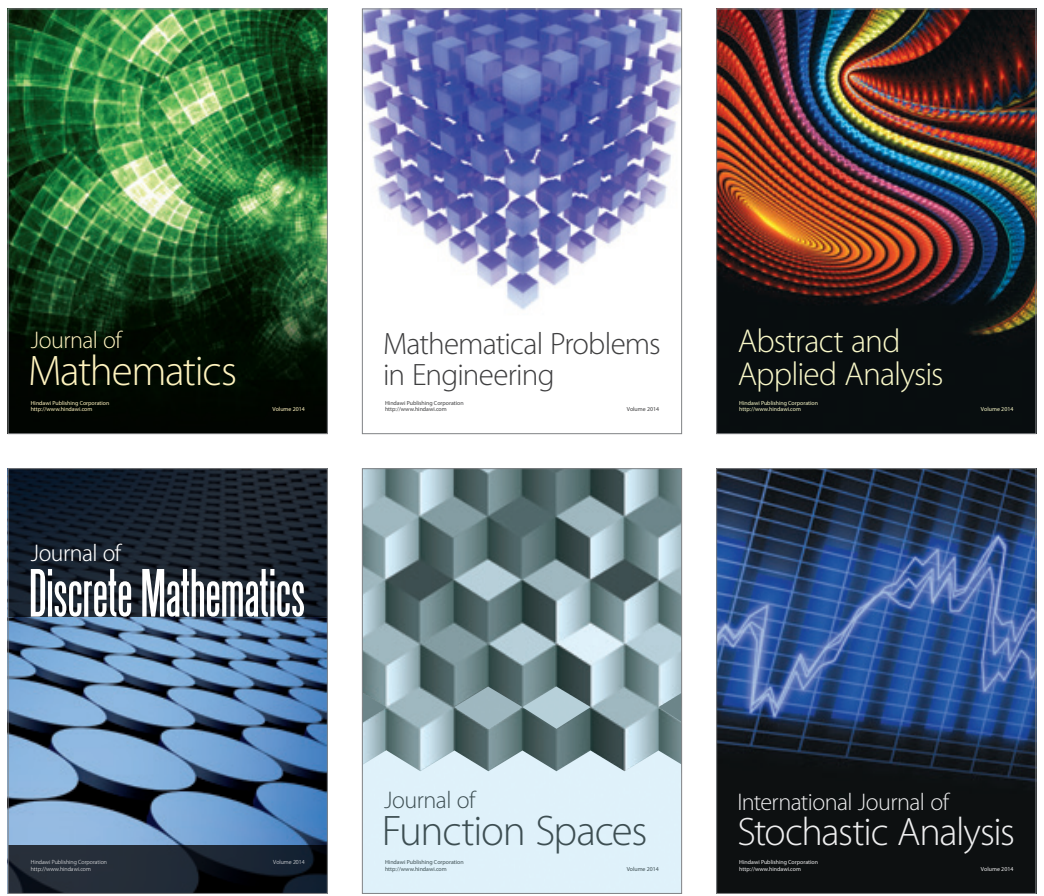

Journal of

Function Spaces

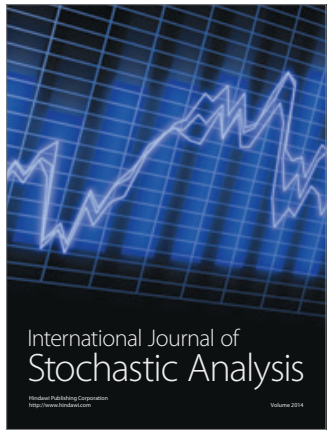

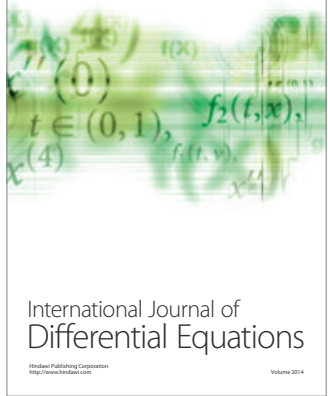
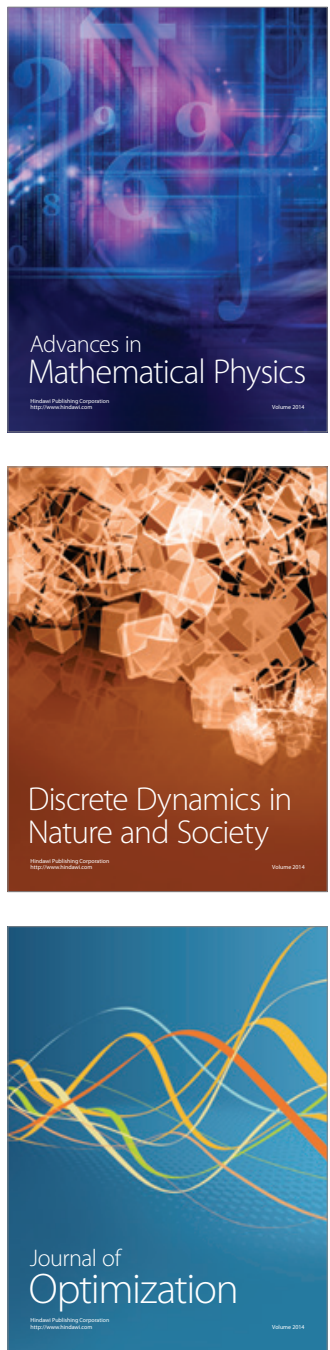